\section{¿POR QUÉ LAS INTERVENCIONES EFECTIVAS DE SUPERVIVENCIA INFANTIL NO LLEGAN A LOS QUE MÁS NECESITAN?}

\section{WHY DO EFFECTIVE INTERVENTIONS TO IMPROVE CHILDHOOD SURVIVAL NOT REACH THOSE WHO MOST NEED THEM?}

Anibal Velásquez ${ }^{1, a}$

Sr. Editor. Las evaluaciones de los Objetivos de Desarrollo del Milenio ${ }^{(1)}$ han demostrado que las intervenciones efectivas que salvan vidas de niños, no llegan a todos aquellos que necesitan; en particular, no llegan a los más pobres. Los programas destinados a ofrecer estas intervenciones son muy a menudo irregulares, de baja calidad, inequitativos y de corta duración ${ }^{(2)}$. Esta situación se explica en parte por las limitaciones y deficiencias en los sistemas de salud y porque se ha prestado poca atención a la implementación de estas intervenciones, en especial, se ha registrado falta de disponibilidad de medios financieros y técnicos y la falta de infraestructura en los países con recursos escasos.

En consecuencia, para expandir las intervenciones en las áreas más pobres es necesario conocer cuáles son las restricciones, su nivel de importancia, así como el grado en que la restricción puede ser tratada o reducida. Las restricciones de un país se pueden identificar en las siguientes dimensiones ${ }^{(3)}$ : i) la comunidad y el hogar, ii) los servicios de salud, iii) la política del sector salud y la gestión estratégica, iv) las políticas públicas intersectoriales y v) las características contextuales (ambientales, la gobernanza y el marco de política general). La comprensión de las limitaciones que enfrentan los países es fundamental para la estimación de las necesidades de recursos para ampliar las intervenciones eficaces (ampliación) y para la toma de decisiones estratégicas sobre las formas de difusión, la secuencia de acciones y la velocidad de la expansión de los servicios.

La OMS ha declarado que será imposible alcanzar los objetivos nacionales e internacionales - incluyendo los Objetivos de Desarrollo del Milenio (ODM) - sin una inversión mayor y más efectiva en los sistemas de salud y servicios ${ }^{(4)}$. Si bien se necesitan más recursos, también se necesita hacer más con los recursos existentes.

1 Abt Associates Inc. Lima, Perú.

a Magister en Malariología y Saneamiento Ambiental.

Recibido: 23-02-11 Aprobado: 09-03-11
Fortalecer los sistemas de salud significa establecer dónde y por qué la inversión es necesaria, establecer los resultados esperados y monitorear el cambio deseado. Los componentes del sistema de salud son: la prestación de servicios, personal de salud, información, productos médicos, vacunas y tecnologías, financiación, y el liderazgo y la gobernanza (gestión). Por esta razón, se recomienda medir la disponibilidad de servicios de salud, la disponibilidad y distribución del personal de salud en las áreas más vulnerables, la capacidad técnica, la capacidad de gestión y supervisión de los programas esenciales o de las intervenciones efectivas, la disponibilidad y acceso a los medicamentos y suministros médicos, el equipamiento y la infraestructura.

Algunas restricciones se pueden aliviar con fondos adicionales, aunque puede ser más difícil para superar los problemas sistémicos. Los recursos financieros pueden mejorar la infraestructura, el equipamiento y los suministros médicos. Sin embargo, puede ser más difícil de reformar los sistemas de gestión de los recursos o el cambio de comportamiento para mejorar la práctica profesional. Del mismo modo, es más difícil superar los problemas de distribución de recursos humanos, la motivación y el rendimiento individual y la capacidad para la planificación y gestión de dichos recursos en el sector salud.

Las políticas y gestión en el sector de la salud también pueden actuar como obstáculos para la ampliación de las intervenciones efectivas. Se describen como restricciones a los sistemas excesivamente centralizados de planificación y gestión; con debilidad de las estructuras de incentivos para utilizar los insumos de manera eficiente y responder a las necesidades de los usuarios, las políticas débiles de sistemas de suministro de medicamentos, la regulación inadecuada de los productos farmacéuticos y proveedores del sector privado, la falta de coordinación entre los sectores, con participación limitada de la sociedad civil, y la dependencia de financiación de los donantes.

En los países con escasos recursos humanos y financieros disponibles y con sistemas de salud con limitaciones, la implementación de las intervenciones efectivas tienden a ser verticales y selectivas. En cambio cuando los sistemas descentralizados de salud están mejor establecidos y la planificación a más largo plazo adoptada, los mecanismos horizontales son más fáciles de implementar.

Aún existe un debate sobre cuál debe ser el mejor enfoque, entre lo vertical y horizontal. Los programas verticales tienden a ofrecer una selección de intervenciones, a menudo de forma independiente, con una gestión 
especializada, la logística y los mecanismos de entrega. En cambio, los programas horizontales tienden a incorporar varias intervenciones de salud como parte de un enfoque integral de atención primaria, por lo general a través de establecimientos de salud del gobierno.

Los programas integrados, además de ser integrales, combinan la gestión y la prevención de una serie de condiciones a nivel individual. Un buen ejemplo es la AIEPI (Atención Integral de las Enfermedades Prevalentes de la Infancia), que se aprovecha de la atención clínica por una enfermedad específica para diagnosticar otras condiciones y para realizar intervenciones preventivas. Podría haber una necesidad de combinar un enfoque vertical en los niveles superiores (por ejemplo, las políticas nacionales y la gestión del programa) con un enfoque horizontal en los niveles inferiores (por ejemplo, manejo de casos y la entrega conjunta de las intervenciones esenciales dirigida a los mismos grupos de población como con la estrategia AIEPI)(5).

También es importante comprender la complejidad de la intervención en cuanto a la calidad y cantidad de recursos necesarios para la expansión de las intervenciones efectivas y su sostenibilidad ${ }^{(6)}$. La complejidad de la intervención depende de la propia intervención, las características de la entrega, los requisitos en términos de capacidad de los gobiernos, y las características de uso. Comprender la complejidad de intervención puede ayudar en la identificación de estrategias para superar las limitaciones de recursos. Las intervenciones complejas son difíciles de implementar en países con sistemas de salud débiles por lo que se necesita apoyar mecanismos de entrega alternativos, como los planes de extensión o los proveedores privados del sector salud.

La salud de los niños depende no solamente de la implementación de intervenciones efectivas sino también de las condiciones sociales y ambientales donde viven. El enfoque de determinantes sociales conduce a desarrollar políticas más equitativas para reducir la pobreza, incrementar la protección social e incrementar el acceso a los servicios de la población más vulnerable. $Y$ esto puede ser posible con el concurso de los otros sectores fuera del sector salud. Los niños son especialmente susceptibles a los factores de riesgo ambientales; pero los niños pobres están más expuestos y son más vulnerables a la mayoría de estos riesgos. Además que el entorno puede causar morbilidad y mortalidad directamente en los niños, de forma indirecta afecta muchas veces impidiendo la implementación y expansión de los programas esenciales y las intervenciones efectivas de salud de la niñez.
En conclusión, las intervenciones efectivas de supervivencia infantil no llegan a los más pobres si no se toman en cuenta las limitaciones y deficiencias de los sistemas de salud. Expandir las intervenciones efectivas exige mayores recursos financieros, humanos y técnicos, mejor equipamiento de los servicios de salud, mayor participación de la comunidad, mejorar la capacidad de administración de los recursos, fortalecer la descentralización del sector salud, implementar programas integrados, implementar acciones para modificar los determinantes, en especial el acceso a agua y saneamiento. Se recomienda que el diseño de la operación y expansión de las intervenciones efectivas en áreas vulnerables incluya el financiamiento del fortalecimiento del sistema de salud y de la articulación con los gobiernos locales y la comunidad. Por la limitación de recursos que existe en la mayoría de países se necesita seleccionar las intervenciones más efectivas e implementarlas con calidad y eficacia.

\section{Conflictos de Interés}

El autor declara no tener conflictos de interés en la publicación del presente artículo.

\section{REFERENCIAS BIBLIOGRÁFICAS}

1. Victora CG, Wagstaff A, Schellenberg JA, Gwatkin D, Claeson M, Habicht JP.Applying an equity lens to child health and mortality: more of the same is not enough.Lancet. 2003;362:233-41.

2. Victora C, Hanson K, Bryce J, Vaughan P. Achieving universal coverage with health interventions.Lancet. 2004;364:1541-48

3. Hanson K, Ranson K, Oliveira-Cruz V, Mills A.Expanding access to priority health interventions: a framework for understanding the constraints to scaling-up. J IntDev 2003;15(1):1-14.

4. Organización Mundial de la Salud. Everybody's business: Strengthening health systems to improve health outcomes WHO's framework for action. Geneva: World Health Organization; 2007.

5. Oliveira-Cruz V, Kurowski C, Mills A.Delivery of priority health services: searching for synergies within the vertical versus horizontal debate.J IntDev2003;15(1):67-86.

6. Gericke CA, Kurowski C, Ranson MK, Mills A.Intervention complexity - a conceptual framework to inform prioritysetting in health. Bulletin of the World Health Organization. 2005;83(4):285-93.

Correspondencia: Anibal Velásquez

Dirección: Av. Del Sur N. ${ }^{\circ} 368$, Urb. Chacarilla. Lima 33, Perú.

Teléfono:(511) 372-7276

Correo electrónico: anibal.velasquez@gmail.com 